\title{
AVALIAÇÃO DO DESEMPENHO DE HIDROCICLONES POR OTIMIZAÇÃO ROBUSTA
}

\author{
V. A. GARCIA ${ }^{1}$, G. S. SILVEIRA ${ }^{1}$, F. S. LOBATO $^{1}$ e L. G. M. VIEIRA ${ }^{1}$ \\ ${ }^{1}$ Universidade Federal de Uberlândia, Faculdade de Engenharia Química \\ E-mail para contato: fslobato@ufu.br
}

\begin{abstract}
RESUMO - No projeto de sistemas de engenharia é comumente necessária a determinação de equações constitutivas (empíricas) para relacionar as variáveis dependentes e independentes em modelos que, não necessariamente, são fundamentados em leis de conservação de massa, energia e momento. Tradicionalmente, durante esse procedimento, deixa-se de considerar que estas equações podem ser sensíveis a pequenas perturbações no vetor de parâmetros de entrada, o que leva a discrepâncias no valor da função objetivo para alguns sistemas. Neste contexto, o presente trabalho tem por objetivo propor, a partir do tratamento de dados experimentais e da aplicação de otimização pelo algoritmo de Evolução Diferencial associada ao conceito de média efetiva, uma metodologia robusta para a determinação de equações constitutivas para o número de Euler, para a razão de líquido e a para a eficiência total empregadas para a avaliação do desempenho de hidrociclones no processo de separação sólido-líquido. Os resultados obtidos demonstram que a metodologia proposta configura-se como uma interessante estratégia, uma vez que foram estimados parâmetros que, sob determinadas condições, são menos sensíveis a pequenas perturbações quando comparados com a solução não robusta.
\end{abstract}

\section{INTRODUÇÃO}

Tradicionalmente, durante a obtenção de equações constitutivas considera-se que o resultado não é afetado por pequenas perturbações no valor dos parâmetros estimados. Contudo, existem casos em que a solução ótima pode ser sensível a mudanças nas variáveis de interesse, sendo necessária a aplicação de otimização robusta para o tratamento deste tipo de problema. Segundo Taguchi (1984), define-se otimização robusta como uma estratégia capaz de produzir uma solução pouco sensível, sob determinadas condições, a pequenas alterações no vetor de variáveis de interesse. É importante ressaltar que uma solução robusta pode não coincidir com a solução nominal, sendo assim a solução robusta tende a ter um resultado "depreciado" em relação à solução nominal.

Diante do que foi exposto, o presente trabalho tem por objetivo aplicar o conceito de Média Efetiva (Deb e Gupta, 2006) associado ao algoritmo de Evolução Diferencial (ED) (Storn e Price, 1995) para a determinação de equações constitutivas empregadas para a avaliação do desempenho de hidrociclones no processo de separação sólido-líquido de um importante minério da região do Alto Paranaíba em Minas Gerais, a saber, a rocha fosfática. Neste caso, deseja-se determinar, através do tratamento de pontos experimentais obtidos por 
Vieira (2006), equações constitutivas para a razão de líquido $\left(R_{L}\right)$, do número de Euler $(E u)$ e da eficiência total $\left(E_{T}\right)$, em função das dimensões geométricas características do hidrociclone.

\section{Evolução Diferencial e Otimização Robusta}

O algoritmo de ED configura-se como uma das principais abordagens para a resolução de problemas de otimização nos dias atuais. Nesta abordagem, a geração de candidatos a solução do problema de otimização é obtida a partir da realização de operações vetoriais simples (soma e subtração) de modo que estes candidatos possam ser avaliados segundo a função objetivo. Neste caso, os parâmetros de controle desta técnica evolutiva são: tamanho da população, taxa de perturbação (que pondera a subtração vetorial), probabilidade de cruzamento e estratégia empregada para a geração dos candidatos (Storn e Price, 1995).

A inserção de robustez no problema de otimização pode ser realizada via aplicação do conceito de Média Efetiva (ME) (Deb e Gupta, 2006): Uma solução $x^{*}$ é denominada solução robusta se a solução ótima é viável para o seguinte problema de otimização, definido em relação à vizinhança $\delta$ (denominado de parâmetro de incerteza) de uma solução $x$ :

$$
\min \frac{1}{\left|\Upsilon_{\delta}(x)\right|} \int_{y \in \Upsilon_{\delta}(x)} f d y
$$

na qual $\left|\Upsilon_{\delta}\right|$ é o hipervolume da vizinhança e $f$ é a função objetivo a ser minimizada. Para a avaliação da integral dada pela Equação (1), um conjunto finito de $H$ soluções deve ser gerado "aleatoriamente" usando, por exemplo, o Hipercubo Latino. Neste caso, definindo-se a vizinhança $\delta$ em relação ao vetor de variáveis de projeto, $N$ soluções $x$ são geradas empregando-se o Hipercubo Latino, sendo a integral avaliada numericamente. Nesta abordagem, minimiza-se a integral referente às perturbações realizadas nas vizinhanças do candidato a solução do problema, ao invés de otimizar-se o próprio candidato.

\section{Hidrociclones}

Os hidrociclones são equipamentos empregados para a separação com grande aplicabilidade em engenharia química. Estes são, basicamente, dotados de uma parte cilíndrica acoplada a uma parte cônica. As dimensões de cada parte (ver Figura 1) são importantes no processo de separação, pois estão diretamente relacionadas com o poder de classificação e a capacidade do equipamento (Vieira, 2006).

Na Figura $1, D_{c}$ é o diâmetro da parte cilíndrica, $D_{o}$ é o diâmetro do overflow, $D_{i}$ é o diâmetro interno do duto de alimentação, $D_{u}$ é o diâmetro do underflow, $L$ o comprimento total do hidrociclone, $l$ é o comprimento da parte do duto de overflow interna ao cilindro e $\theta$ é o ângulo da parte cônica. Para fins de operação, a suspensão é alimentada tangencialmente na parte cilíndrica. Há ainda um tubo localizado axialmente na parte superior que é por onde a suspensão diluída (overflow), dotada de partículas finas, é coletada. Já a parte cônica possui um orifício inferior, que é o responsável pelo direcionamento da suspensão concentrada (underflow), constituída de partículas maiores. 
Em se tratando de hidrociclones, o trinômio energia-concentração-classificação deve ser sempre observado no que tange à viabilidade da operação unitária de hidrociclonagem, sendo estes quantificados, respectivamente, pelo Número de Euler $(E u)$, Razão de Líquido $\left(R_{L}\right)$ e Eficiência Total $\left(E_{T}\right)$. Sendo assim, para o projeto deste equipamento faz-se necessária a determinação de equações constitutivas que relacionam estas variáveis dependentes $\left(E u, R_{L} \mathrm{e}\right.$ $\left.E_{T}\right)$ com as dimensões geométricas do separador.

Figura 1 - Principais dimensões geométricas de um hidrociclone (Vieira, 2006).

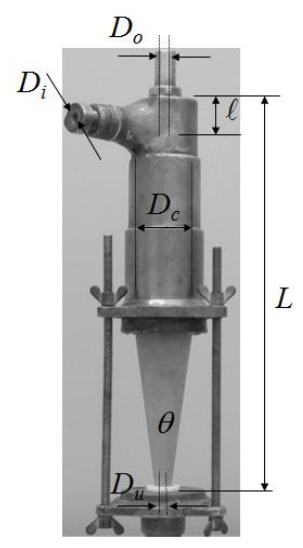

\section{Formulação do Problema de Otimização}

Para a determinação do trinômio citado anteriormente, deve-se propor e resolver um problema de otimização. Este consiste na minimização do funcional $f$, isto é, obter o valor do vetor de variáveis de projeto de modo a minimizar a diferença entre os valores experimentais e preditos pelo modelo, conforme a seguinte equação:

$$
f \equiv \frac{\sum_{i=1}^{n_{\text {exp }}}\left(y_{i}^{\exp }-y_{i}^{\text {cal }}\left(\beta_{1}, \beta_{2}, \ldots, \beta_{m}\right)\right)^{2}}{\left(y_{\max }^{\exp }\right)^{2}}
$$

Em que $y^{\text {cal }}$ e $y^{\text {exp }}$ representam o valor da variável dependente $(y)$ predito pelo modelo e o seu correspondente experimental, respectivamente. $\beta_{k}(k=1, \ldots, m)$ é o vetor que contém os $m$ parâmetros que devem ser determinados, $n_{\exp }$ é o número de dados experimentais considerados no ajuste do modelo e $y_{\max }^{\exp }$ é o maior valor experimental observado para a resposta considerada na análise.

\section{Resultados e Discussão}

Para a resolução do problema proposto alguns pontos devem ser destacados:

1. As informações experimentais utilizadas para a determinação de cada equação constitutiva $\left(R_{L}, E u\right.$ e $\left.E_{T}\right)$ foram obtidas por Vieira (2006), que estudou o efeito das principais dimensões geométricas de um hidrociclone, mediante utilização de 25 diferentes hidrociclones e rocha fosfática como material particulado (ver a Tabela 1). 
2. As respostas analisadas foram obtidas a partir do estudo dos efeitos exercidos pelas seguintes relações (Vieira, 2006): $X_{1}=2 / 0,1\left(D_{i} / D_{c^{-}}-0,21\right) ; \quad X_{2}=2 / 0,1\left(D_{o} / D_{c^{-}}-0,27\right)$; $X_{3}=2 / 2,16\left(L / D_{c^{-}} 5,8\right)$ e $X_{4}=2 / 6,62(\theta-0,21)$.

3. Para a determinação dos parâmetros em cada uma das equações constitutivas, foi considerada a seguinte lei de formação (em que $\varepsilon$ é o erro cometido):

$$
Y=\beta_{o}+\sum_{i=1}^{k} \beta_{i} x_{i}+\sum_{i i=1}^{k} \beta_{i i} x_{i}^{2}+\sum_{i<j} \sum_{i}^{k} \beta_{i j} x_{i} x_{j}+\varepsilon
$$

Tabela 1 - Planejamento Composto Central com 5 réplicas no centro (Vieira, 2006).

\begin{tabular}{|c|c|c|c|c|c|c|c|c|c|c|c|c|c|c|c|}
\hline & $X_{1}$ & $X_{2}$ & $X_{3}$ & $X_{4}$ & $E u$ & $\begin{array}{c}R_{L} \\
(\%)\end{array}$ & $\begin{array}{c}E_{T} \\
(\%)\end{array}$ & & $X_{1}$ & $X_{2}$ & $X_{3}$ & $X_{4}$ & $E u$ & $\begin{array}{c}R_{L} \\
(\%)\end{array}$ & $\begin{array}{c}E_{T} \\
(\%)\end{array}$ \\
\hline $\mathrm{H}_{1}$ & -1 & -1 & -1 & -1 & 4429 & 28,96 & 62,54 & $\mathrm{H}_{16}$ & +1 & +1 & +1 & +1 & 937 & 17,10 & 55,31 \\
\hline $\mathrm{H}_{2}$ & -1 & -1 & -1 & +1 & 4200 & 28,75 & 59,46 & $\mathrm{H}_{17}$ & $-\alpha$ & 0 & 0 & 0 & 6207 & 16,45 & 60,41 \\
\hline $\mathrm{H}_{3}$ & -1 & -1 & +1 & -1 & 3801 & 32,35 & 70,40 & $\mathrm{H}_{18}$ & $+\alpha$ & 0 & 0 & 0 & 1096 & 19,32 & 59,27 \\
\hline $\mathrm{H}_{4}$ & -1 & -1 & +1 & +1 & 3624 & 31,55 & 60,12 & $\mathrm{H}_{19}$ & 0 & $-\alpha$ & 0 & 0 & 2803 & 43,20 & 76,00 \\
\hline $\mathrm{H}_{5}$ & -1 & +1 & -1 & -1 & 3588 & 11,07 & 54,01 & $\mathrm{H}_{20}$ & 0 & $+\alpha$ & 0 & 0 & 1666 & 13,05 & 66,56 \\
\hline $\mathrm{H}_{6}$ & -1 & +1 & -1 & +1 & 3196 & 10,98 & 46,67 & $\mathrm{H}_{21}$ & 0 & 0 & $-\alpha$ & 0 & 2589 & 16,22 & 54,56 \\
\hline $\mathrm{H}_{7}$ & -1 & +1 & +1 & -1 & 3175 & 15,76 & 60,94 & $\mathrm{H}_{22}$ & 0 & 0 & $+\alpha$ & 0 & 1508 & 20,65 & 48,21 \\
\hline $\mathrm{H}_{8}$ & -1 & +1 & +1 & +1 & 2900 & 18,08 & 53,00 & $\mathrm{H}_{23}$ & 0 & 0 & 0 & $-\alpha$ & 2137 & 20,80 & 61,73 \\
\hline $\mathrm{H}_{9}$ & +1 & -1 & -1 & -1 & 2365 & 29,41 & 71,69 & $\mathrm{H}_{24}$ & 0 & 0 & 0 & $+\alpha$ & 1773 & 18,00 & 60,70 \\
\hline $\mathrm{H}_{10}$ & +1 & -1 & -1 & +1 & 1768 & 32,77 & 68,12 & $\mathrm{H}_{25}$ & 0 & 0 & 0 & 0 & 1847 & 18,03 & 62,90 \\
\hline $\mathrm{H}_{11}$ & +1 & -1 & +1 & -1 & 1502 & 32,80 & 70,00 & $\mathrm{H}_{25}$ & 0 & 0 & 0 & 0 & 1858 & 17,67 & 61,98 \\
\hline $\mathrm{H}_{12}$ & +1 & -1 & +1 & +1 & 1439 & 31,98 & 63,64 & $\mathrm{H}_{25}$ & 0 & 0 & 0 & 0 & 1889 & 17,94 & 66,08 \\
\hline $\mathrm{H}_{13}$ & +1 & +1 & -1 & -1 & 1656 & 10,90 & 67,67 & $\mathrm{H}_{25}$ & 0 & 0 & 0 & 0 & 1804 & 17,87 & 68,56 \\
\hline $\mathrm{H}_{14}$ & +1 & +1 & -1 & +1 & 1170 & 14,73 & 58,64 & $\mathrm{H}_{25}$ & 0 & 0 & 0 & 0 & 1902 & 18,09 & 57,85 \\
\hline $\mathrm{H}_{15}$ & +1 & +1 & +1 & -1 & 1040 & 17,25 & 60,35 & & & & & & & & \\
\hline
\end{tabular}

*Réplicas no centro. a (nível extremo do planejamento) igual a 1,66.

4. Parâmetros usados pelo algoritmo de ED: 100 indivíduos; 1000 gerações; estratégia 7 (Storn e Price, 1995) para a geração de candidatos; probabilidade de cruzamento e taxa de perturbação iguais a 0,8 . O algoritmo foi executado 10 vezes para a obtenção dos melhores valores que são apresentados a seguir. Espaço de projeto: $-2500<\beta_{i}$ $<2500(i=1, \ldots, 15)$.

5. Análise de incerteza: parâmetro de incerteza $\delta$ igual a 0 (solução nominal) e 0,25 (solução robusta). Para cada uma das variáveis de projeto gerou-se um domínio definido a partir da variável desvio $\delta$. Para o cômputo numérico da integral via método dos trapézios, foram consideradas 100 amostras geradas pelo Método de Monte Carlo. É importante ressaltar que para o caso nominal são necessárias 100+100×1000 avaliações da função objetivo em cada execução. Já para o caso robusto, são necessárias $100+100 \times 1000 \times 100$ avaliações da função objetivo. 
6. Para mensurar a qualidade da solução obtida com a estimação dos parâmetros foi utilizado o coeficiente de determinação $\left(r^{2}\right)$.

Na Tabela 2 são apresentados os coeficientes das equações constitutivas para $E u, R_{L}$ e $E_{T}$ estimados pelo algoritmo de ED considerando $\delta=0$ e $\delta=0,25$. Para o caso nominal, observou-se que os resultados obtidos (função objetivo e coeficiente de determinação) estão em concordância com aqueles obtidos por Vieira (2006). Quando um determinado nível de incerteza foi inserido na estimação dos parâmetros, notou-se um aumento no valor da função objetivo e uma diminuição do coeficiente de determinação. Este resultado já era esperado, uma vez que, na prática, a solução robusta dificilmente coincidirá com a solução nominal. Esta depreciação do valor do coeficiente de determinação fica mais evidente no caso da eficiência total.

Tabela 2 - Coeficientes estimados pelo algoritmo de ED para $E u, R_{L}$ e $E_{T}$.

\begin{tabular}{|c|c|c|c|c|c|c|}
\hline \multirow{2}{*}{} & \multicolumn{2}{|c|}{$E u$} & \multicolumn{2}{c|}{$R_{L}$} & \multicolumn{2}{c|}{$E_{T}$} \\
\cline { 2 - 7 } & $\delta=0$ & $\delta=0,25$ & $\delta=0$ & $\delta=0,25$ & $\delta=0$ & $\delta=0,25$ \\
\hline$\beta_{0}$ & 1909,1848 & 1794,6458 & 17,8049 & 16,5044 & 63,0228 & 56,9888 \\
\hline$\beta_{1}$ & $-1186,3213$ & $-1183,7777$ & 0,6460 & 0,4762 & 2,1708 & 1,8984 \\
\hline$\beta_{2}$ & $-341,8753$ & $-344,5826$ & $-8,5205$ & $-8,4855$ & $-3,9706$ & $-4,4199$ \\
\hline$\beta_{3}$ & $-267,2235$ & $-226,2580$ & 1,6960 & 1,5821 & $-0,2722$ & 0,0579 \\
\hline$\beta_{4}$ & $-136,0269$ & $-117,9473$ & 0,1245 & 0,0847 & $-2,5081$ & $-2,4901$ \\
\hline$\beta_{11}$ & 596,4587 & 611,8303 & 0,1113 & 0,7108 & $-0,8505$ & 1,0364 \\
\hline$\beta_{22}$ & 82,2324 & 131,2364 & 3,8216 & 3,7839 & 3,3156 & 4,3040 \\
\hline$\beta_{33}$ & 14,7152 & 69,2753 & 0,2873 & 0,7869 & $-3,9470$ & $-2,1416$ \\
\hline$\beta_{44}$ & $-19,2061$ & 1,1194 & 0,6487 & 0,8051 & $-0,3768$ & 1,7107 \\
\hline$\beta_{12}$ & 57,7519 & 43,3238 & $-0,1074$ & $-0,3060$ & 0,3926 & $-0,1772$ \\
\hline$\beta_{13}$ & $-8,0117$ & $-4,2480$ & $-0,4408$ & $-0,3052$ & $-2,4222$ & $-3,0854$ \\
\hline$\beta_{14}$ & $-10,9927$ & $-9,2216$ & 0,3188 & 0,2139 & 0,2808 & 0,2877 \\
\hline$\beta_{23}$ & 52,3677 & 15,9032 & 0,7142 & 0,8254 & 0,0389 & 0,2591 \\
\hline$\beta_{24}$ & $-11,9019$ & $-43,7644$ & 0,2839 & 0,3488 & $-0,3674$ & $-0,0700$ \\
\hline$\beta_{34}$ & 67,8519 & 84,8600 & $-0,4092$ & $-0,1204$ & $-0,4542$ & $-0,4431$ \\
\hline$f$ & 0,0289 & 0,0993 & 0,0103 & 0,1205 & 0,0360 & 0,4068 \\
\hline$r^{2}$ & 0,9732 & 0,9687 & 0,9898 & 0,9802 & 0,8397 & 0,6529 \\
\hline
\end{tabular}

Para avaliar a influência do parâmetro de incerteza no valor da função objetivo obtida para a eficiência total foram gerados e avaliados 1000 pontos aleatórios em cada uma das soluções (nominal e robusta) encontradas considerando o seguinte vetor de incertezas $[0,00$ $0,010,050,100,250,50]$. Neste caso, a média $(\Omega(f))$ e o desvio padrão $(\Phi(f))$, em relação à função objetivo, puderam ser determinados, de acordo com o apresentado na Figura 2.

Nestas figuras, quando o valor de $\delta$ foi incrementado para avaliar o seu efeito sobre a solução nominal, constatou-se uma maior sensibilidade em relação à solução robusta correspondente, conforme os valores computados para a média e o desvio padrão. Assim, verificou-se que a solução robusta foi menos sensível à perturbações quando comparada a sua correspondente nominal, justificando a utilização de uma abordagem específica para a avaliação da presença de incertezas no vetor de variáveis de projeto. Em termos do número de avaliações da função objetivo, observou-se que a metodologia robusta requer mais avaliações 
do que a correspondente nominal. Este resultado deve-se às avaliações necessárias para a determinação da integral.

Figura 2 - Média e desvio padrão da função objetivo em função do parâmetro de incerteza $(\delta)$ para $E_{T}$.
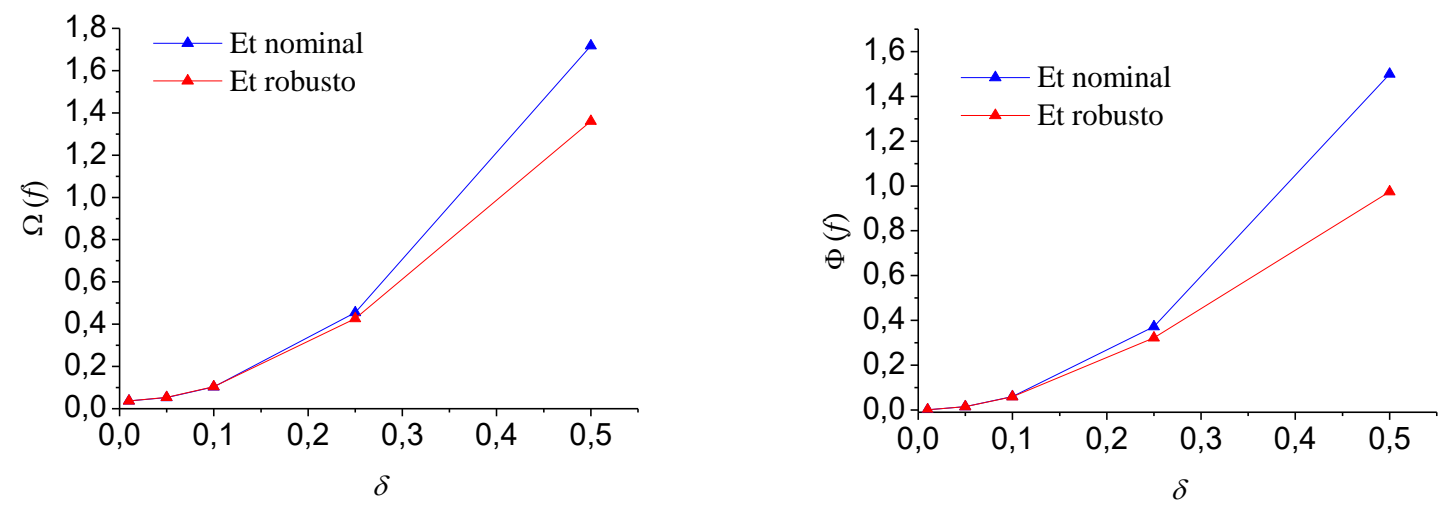

\section{Conclusão}

Este trabalho objetivou o desenvolvimento de uma estratégia sistemática para a determinação de equações constitutivas utilizadas para avaliação do desempenho de hidrociclones através do algoritmo de ED associado ao conceito de ME. Para essa finalidade considerou-se como objetivo a minimização dos erros entre os pontos experimentais e preditos para cada equação constitutiva, através da determinação das dimensões geométricas do hidrociclone. Para cada equação considerada foi analisada a influência do parâmetro de incerteza na qualidade da solução obtida em termos do valor da função objetivo e do coeficiente de determinação. De forma geral observou-se, para os estudos de casos propostos, que a metodologia apresentada configurou-se como uma alternativa interessante para a determinação de equações constitutivas robustas, já que, por exemplo, para a eficiência total, foi obtida uma solução que apresenta menor sensibilidade a pequenas perturbações do sistema em relação à respectiva solução nominal.

\section{REFERÊNCIAS}

DEB, K.; GUPTA, H. Introducing Robustness in Multi-Objective Optimization. Evolutionary Computation, vol. 14, p. 463-494, 2006.

STORN, R.; PRICE, K. Differential Evolution: A Simple and Efficient Adaptive Scheme for Global Optimization over Continuous Spaces. International Computer Science Institute, vol. 12, pp. 1-16, 1995.

TAGUCHI, G. Quality Engineering through Design Optimization. Kraus International Publications. New York, 1984.

VIEIRA, L. G. M.. Otimização dos Processos de Separação em Hidrociclones Filtrantes, Tese de Doutorado, Universidade Federal de Uberlândia, 2006. 